\title{
Successful Revascularization of a Completely Occluded Right Coronary Artery by Local Thrombus Fragmentation, Thrombolysis, Thrombus Aspiration, and Balloon Angioplasty in a Patient with Atypical Kawasaki Disease
}

\section{A Case Report and Review of the Literature}

\author{
Zora Meyer, MD'1, Kai Thorsten Laser, MD, PhD', Sissi Bach, MD'1, Carola Hesse, MD'1, Deniz Kececio- \\ glu, MD, PhD', Werner Scholtz, MD², Christoph M. Happel, MD, PhD', Nikolaus A. Haas, MD, PhD ${ }^{1,3^{*}}$ \\ ${ }^{1}$ Department for Congenital Heart Defects, Heart and Diabetes Centre North Rhine Westphalia, Ruhr University Bochum, \\ Bad Oeynhausen, Germany \\ ${ }^{2}$ Department for Cardiology, Heart and Diabetes Centre North Rhine Westphalia, Ruhr University Bochum, Bad Oeynhausen, Germany \\ ${ }^{3}$ Department for Pediatric Cardiology and Intensive Care, Ludwig Maximilians University Munich, Muenchen, Germany
}

\begin{abstract}
Background: The clinical presentation of atypical Kawasaki disease (KD) is variable; thus, an accurate diagnosis may be missed. With intravenous immunoglobulin therapy, the risk of coronary arterial lesions has been reduced from $20-25 \%$ to about $5 \%$. Coronary artery aneurysms may remain clinically silent, but thrombosis may result in acute myocardial infarction. We report a case with complete occlusion of the right coronary artery (RCA) due to thrombosis of large aneurysms and severe stenoses after atypical KD.

Methods: A 10-year-old boy was admitted to our tertiary medical center after two episodes of ventricular fibrillation caused by acute myocardial infarction.

Results: Coronary angiography showed aneurysms of the left coronary artery and a completely occluded RCA. Transcatheter revascularization was achieved by a combination of mechanical thrombus fragmentation, intracoronary thrombolysis, thrombus aspiration, and balloon angioplasty of two stenosed areas of the RCA,
\end{abstract}

resulting in complete reperfusion. The child's past medical history revealed the diagnosis of untreated atypical KD 6 months previously.

Conclusions: There are few reports of coronary interventions after KD in young patients. Coronary artery abnormalities include persistent aneurysms with the risk of thrombosis and progressive stenosis. However, no confirmed treatment guidelines exist for this particular patient group. Based on the highly variable anatomy of the coronary arteries, an individualized therapy using the full armamentarium of endovascular treatments may be necessary. This case study suggests that primary percutaneous coronary intervention using a targeted approach might be safe and effective in the treatment of acute myocardial infarction after KD.

Copyright @ 2016 Science International Corp.

Key Words

Atypical • Kawasaki disease - Coronary occlusion • Intracoronary lysis • Pediatrics interventions (c) 2016 Journal of Structural Heart Disease Published by Science International Corp. ISSN 2326-4004

Fax +1 2037853346
E-Mail: jshd@scienceinternational.org

http://structuralheartdisease.org/
Accessible online at:

http://structuralheartdisease.org/
* Corresponding Author:

Nikolaus A Haas, MD, PhD

Department for Pediatric Cardiology and Intensive Care

Ludwig Maximilians University

Marchioninistrasse 15, 81377 Muenchen, Germany

Tel.: +49 894400 73941; Fax: +49 89-4400-73943; E-Mail: Nikolaus.Haas@med.uni-muenchen.de 


\section{Introduction}

Kawasaki disease (KD) is an acute febrile disease of unknown origin, characterized by systemic vascular inflammation involving small and medium arteries, with predilection for coronary arteries. Since the first report in 1967 from Japan [1], KD has become the most common form of pediatric systemic vasculitis. The clinical presentation of KD is variable but classically consists of 5 days of fever, accompanied by nonpurulent conjunctivitis, rash on the trunk, erythema of the lips or oral cavity, erythema of hands or feet, and cervical lymphadenopathy. An atypical presentation is difficult to recognize; it may lead to delayed treatment and is thus associated with a higher incidence of coronary aneurysms (CAAs). Immediate treatment with immunoglobulins reduces the incidence of coronary artery aneurysms in children from $25 \%$ to only $5 \%$ [1].

Nowadays, CAAs following KD are the leading cause of acquired heart disease in children and young adults in western countries. Coronary artery abnormalities include persistent aneurysms with the risk of thrombosis and progressive stenosis, with or without the development of extensive collateral circulation. CAAs can persist, progress to stenosis, and lead to acute myocardial infarction [2]. There are few reports of coronary interventions after KD performed in young patients [3]; additionally, due to the highly variable anatomy of the lesions of the coronary arteries after KD, no confirmed standardized treatment guidelines for this particular patient group exist.

Here we report a case of successful and complete revascularization of thrombosed giant aneurysms of the right coronary artery (RCA) including dilatation of severe stenosis in a patient after atypical KD.

\section{Case Presentation}

A 10-year-old male child was transferred to our hospital with the presumed diagnosis of acute myocardial infarction. He suffered from two episodes of ventricular fibrillation that were successfully treated by resuscitation and defibrillation. The electrocardiogram (ECG) showed ST-segment elevation in V1 to V4 and was consistent with myocardial ischemia. The laboratory studies showed an elevated troponin I $(11,232 \mathrm{pg} / \mathrm{ml}$, Ref. 0-26; Figure 1). All other laboratory parameters were normal, including inflammatory markers. Transthoracic echocardiography showed dilatation of the central coronary arteries
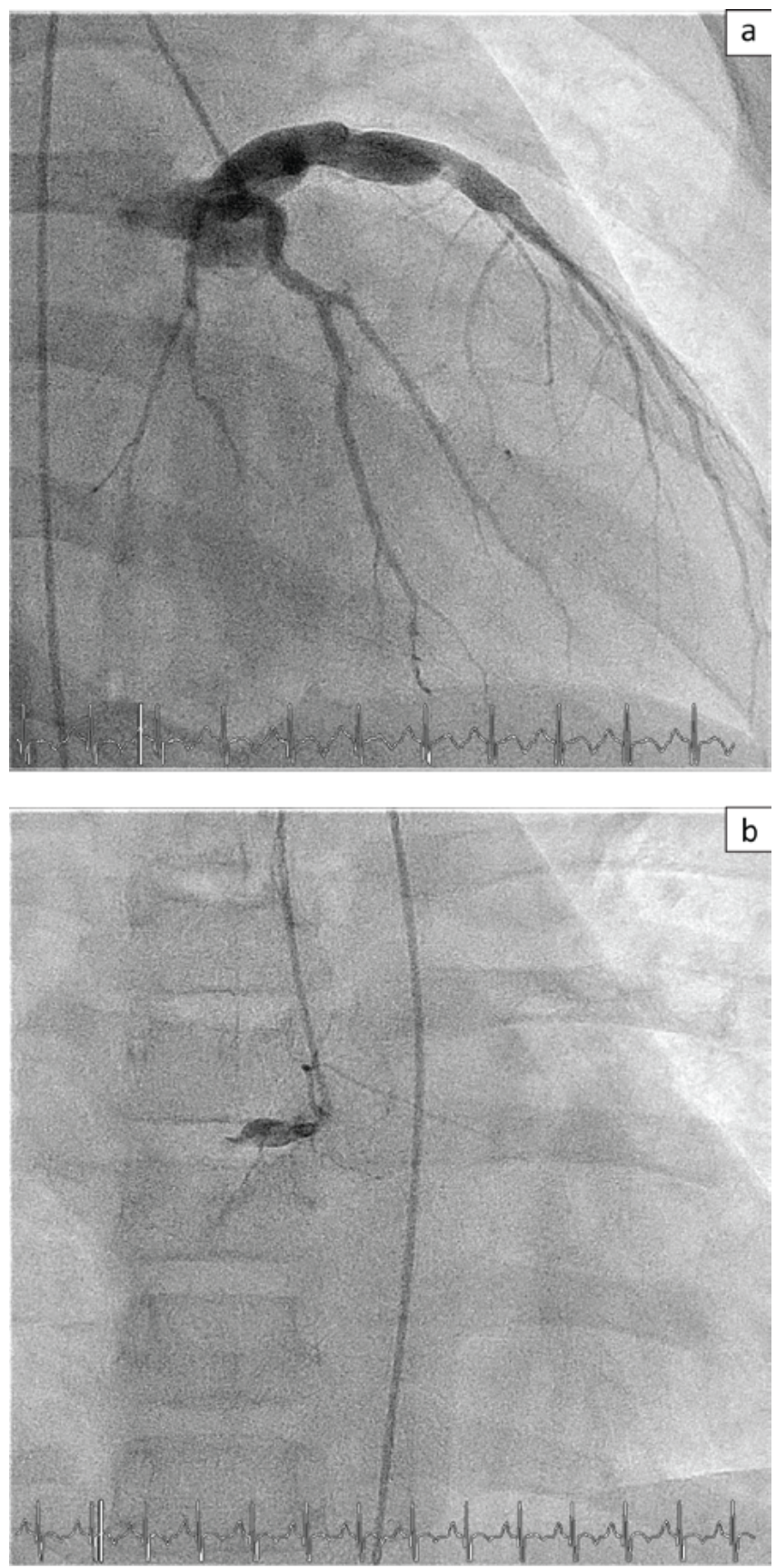

Figure 1. Panel A. Selective coronary angiography of the left and right coronary arteries. Panel $B$. The left coronary artery shows moderate aneurysmatic dilatation of the left anterior descending artery. The right coronary artery is completely occluded (both in strict a. p. $0^{\circ}$ projection). 
and hypokinesia of the posterior wall.

Subsequent coronary angiography showed a left coronary artery with formation of a moderate sized aneurysm, with preserved patency and no intracoronary thrombus formation. The RCA however was completely occluded (Figure 1A and B). We immediately attempted to revascularize the presumably thrombosed RCA. A 0.0018 " coronary guide wire (SV-8, Cordis) was repeatedly advanced into the ostium of the RCA and could be advanced several millimeters. Thereafter, intracoronary thrombolysis was started with tissue-plasminogen activator ( $r T-P A)$ injections at aliquot doses of 1-2 mg. This led to partial thrombolysis and improved visualization of the RCA, showing a large aneurysm filled with thrombotic material (Figure 2A and B). Repetitive direct intracoronary thrombolysis in combination with mechanical thrombus fragmentation using the guide wire and direct thrombus aspiration over the $6 \mathrm{~F}$ coronary guide catheter finally resulted in complete visualization of the RCA (Figure 2C), revealing two large aneurysms in combination with two severe stenoses with a diameter of less than $1 \mathrm{~mm}$ between them. The stenoses were dilated with a 3-mm coronary balloon (Savy, Cordis) at a maximum pressure of 6 atmospheres to improve coronary perfusion (Figure 2D and $\mathrm{E})$. After a total dose of $20 \mathrm{mg}$ intracoronary $\mathrm{rT}$-PA, complete reperfusion of the RCA was achieved (Figure $2 \mathrm{~F}$ ). After complete revascularization, the exact anatomy of the RCA became evident, showing two giant aneurysms combined with severe stenosis (Figure 3). A coronary stent was not used due to the anatomy of the RCA, to avoid both malpositioning of the stent in the aneurysm and the risk of acute secondary thrombotic occlusion. The patient was transferred to the PCICU, and a combination therapy of clopidogrel (75 mg), acetylsalicylic acid (100 mg), and heparinization was administered for the subsequent three days. Control echocardiography on days 2 and 3 confirmed full recovery of the ventricular function. Troponin I levels decreased to normal (Figure 4). A scheduled recatheterization 3 days later showed complete and full restoration of coronary perfusion. The ECG normalized, showing normal function of the left ventricle without dyskinetic areas or apparent scarring. The patient recovered completely and was discharged with a combination therapy of acetylsalicylic acid and war- farin (target INR between 2.0 and 3.0). Planned control catheter evaluations after 6 and 12 months revealed excellent cardiac function and a patent RCA without new thrombus formation.

Despite an initial lack of evidence, the child's past medical history revealed a possible diagnosis of untreated atypical KD 6 months previously. He presented to another hospital with acute febrile illness for about 2 weeks, but with no signs of conjunctivitis, rash, mucosal changes, or lymphadenopathy. After 2 weeks, the fever disappeared and desquamation of the palms was visible. Echocardiography performed on days 4 and 10 of the illness was unremarkable. Follow-up echocardiography was advised, but the patient did not present to a pediatric cardiologist for follow-up examination.

\section{Discussion}

$\mathrm{KD}$ is one of the most important causes of acute coronary syndrome in young adults. About $5 \%$ of all patients with KD develop ischemic heart disease during long-term follow up, which is often associated with calcified stenosis $[4,5]$. Catheter intervention is now established as a first-line therapeutic strategy for adult patients with coronary artery disease, and has provided satisfactory therapeutic results [6]. However, in adult as well as pediatric patients with KD, limited experience with catheter interventions has been reported $[4,7,8]$. Options for coronary revascularization in KD generally consist of intravenous coronary thrombolysis, percutaneous coronary intervention, or coronary artery bypass grafting $[9,10,11,12]$. The guidelines for catheter interventions in KD, published by the research committee of the Japanese Ministry of Health, Labor, and Welfare, indicate that patients with acute myocardial infarction after KD can be candidates for percutaneous transluminal coronary revascularization and intravenous thrombolysis, patients with stenotic lesions with mild calcification can be candidates for percutaneous transluminal coronary balloon angioplasty (PCBA), and patients with severe calcifications can be candidates for rotational ablation [13].

Primary percutaneous coronary interventions in children presenting with clinical symptoms of acute myocardial infarction due to the sequelae of KD have been extremely limited and restricted primarily to 

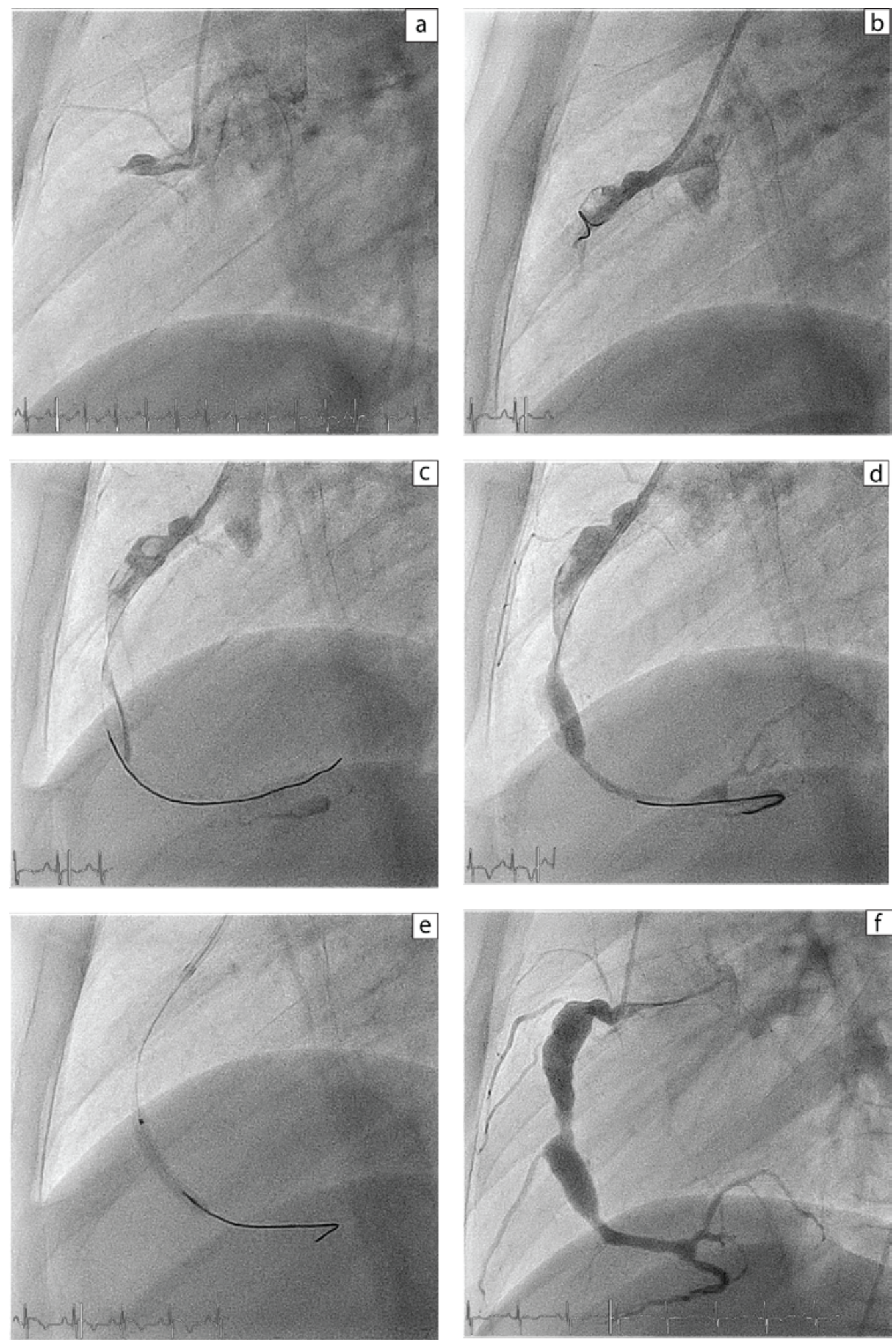

Figure 2. Panel $A$. Selective coronary angiography of the right coronary artery. Panel B. Complete occlusion. Panel C. Mechanical recanalization. Panel D. Local lysis. Panel E. Thrombus aspiration. Panel F. Balloon dilatation of the distal stenosis (all strict lateral $90^{\circ}$ projection). 

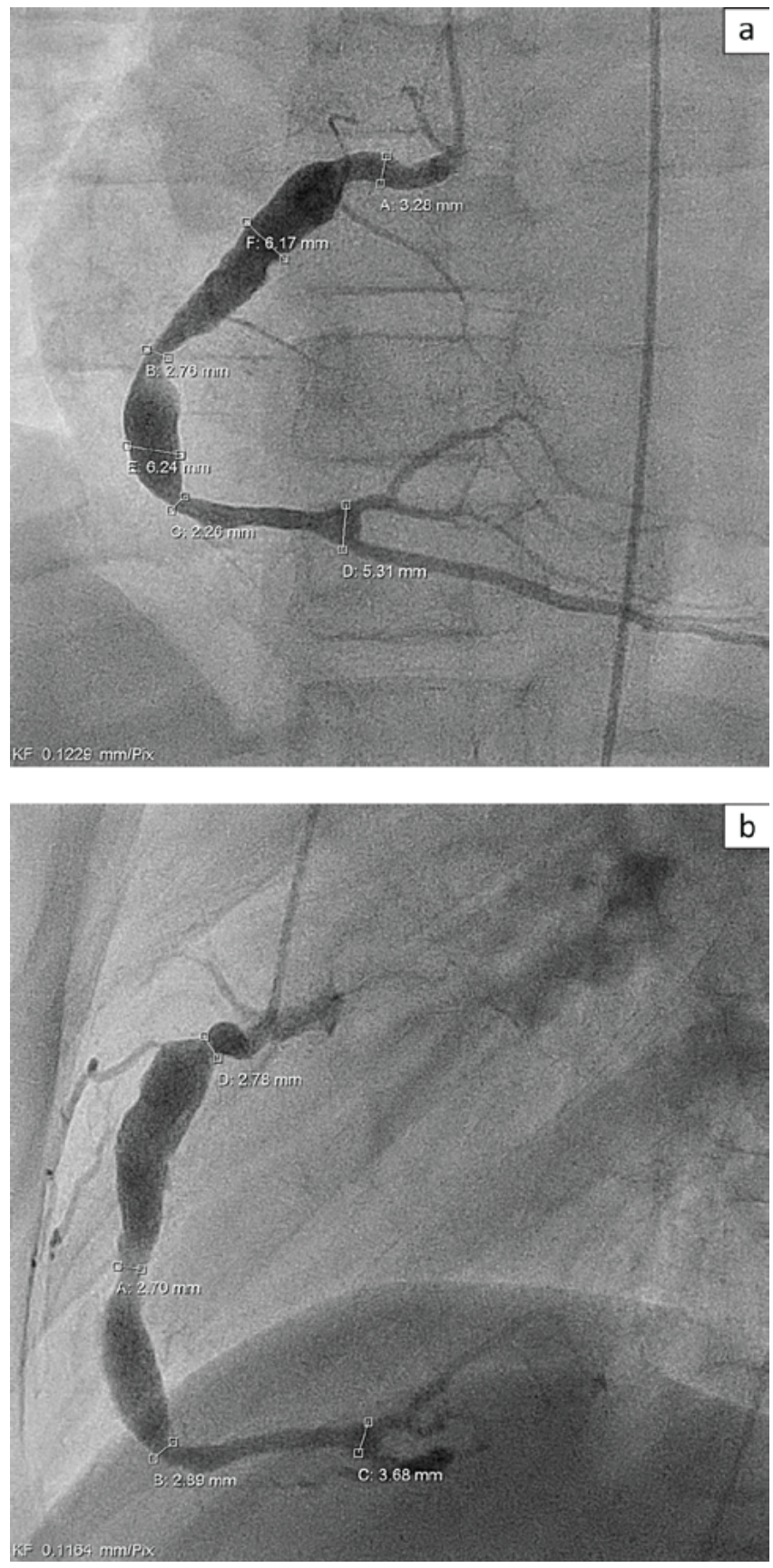

Figure 3. Selective coronary angiography of the right coronary artery (RCA) after complete revascularization Panel A. Frontal view. Panel $B$. Lateral view. The RCA shows two gigantic aneurysms measuring more than $6 \mathrm{~mm}$ in diameter. After dilatation mild stenosis at the exits of the aneurysms persists.

cases from Japan and other Asian countries. Thrombolytic agents such as urokinase and rT-PA have been used to treat acute myocardial ischemia secondary to

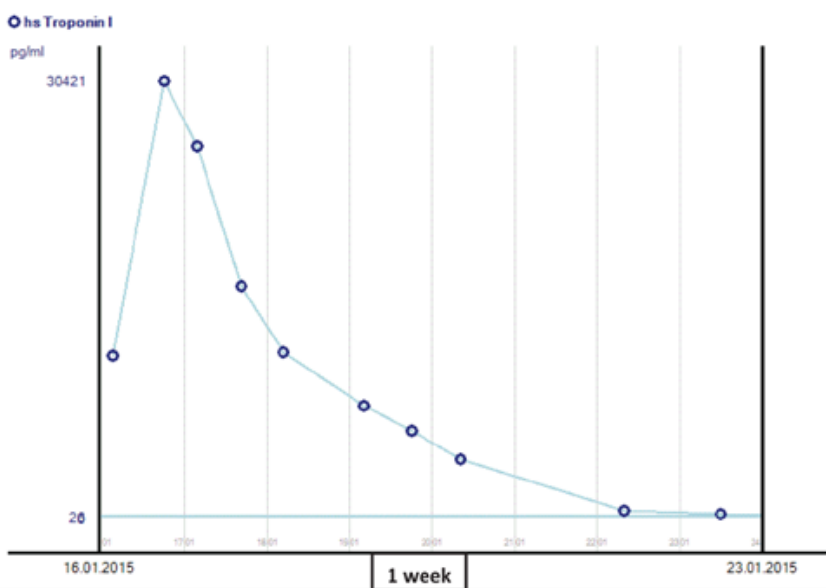

Figure 4. Levels of troponin I after revascularization of the right coronary artery.

thrombus formation in CAAs of patients with KD $[5,10,14]$. Improved outcome was reported when the agents were administered directly into the coronary arteries [5, 15]. Ariyoshi et al. [4] reported three cases, in patients aged $20-35$ years, in whom primary percutaneous coronary interventions in combination with intracoronary thrombolysis proved to be safe and effective in acute myocardial infarction. Karia et al. [14] reported a 29-day-old child with KD who presented with multiple medium-size CAAs as well as coronary thromboses, myocardial ischemia, and congestive heart failure. The infant successfully underwent intracoronary infusion of rT-PA. He had a history of KD at the age of 8 days. Mongiovi et al. [16] reported a 7-year-old boy with acute myocardial infarction in whom primary recanalization with angioplasty and complete reperfusion of the occluded artery was achieved. At 2-year follow up, ventricular function was still normal. Inaba used pulse infusion of thrombolysis (rT-PA) into the thrombus in a 24-year-old man with complete distal thrombosis of the RCA [15].

Interventional treatment in addition to thrombolysis was described in some other studies. Akagi et al. [3] reported the results of a nationwide review of 55 institutions in Japan: In 57 patients, percutaneous transluminal angioplasty (PTCA) was performed in 34, percutaneous transluminal coronary rotational ablation (PTCRA) in 13, directional coronary atherectomy (DCA) in 4, and stent implantation in 7 . The success rate was $74 \%$ for PTCA, $100 \%$ for PTCRA, $100 \%$ for DCA, and $86 \%$ for stents. Ishii et al. [13] reported a 
case series of 23 stenotic lesions in 22 patients (aged 2 to 24 years) where a total of 21 lesions (91\%) could be treated successfully by PTCA, PTCRA, and stent implantation. In general, localized stenosis of the coronary arteries can be treated by balloon angioplasty, and in those patients with severe calcification, PTCRA is the treatment of choice with good short-term results [16-19]. Drossner et al. [20] reported a 3-yearold girl with acute myocardial infarction and large left and RCA aneurysms who had been diagnosed with $\mathrm{KD}$ at 4 months of age. Successful coronary revascularization was performed by balloon angioplasty and placement of two stents. Dahdah et al. [21] reported an $11 \frac{1}{2}$-year-old girl in whom successful recanalization was achieved using a high-frequency mechanical vibration catheter (Crosser catheter); additional balloon dilatation was performed and a stent was deployed. Finally, Parsa et al. [7] reported a 35-year-old man with acute myocardial infarction after a history of KD at the age of 5 years. The patient underwent thrombectomy and a stent was deployed. Grade III flow was established.

Rarely, covered stents may be needed to cover large aneurysms prone to intracoronary thrombus formation. Waki et al. [22] reported satisfactory longterm outcome for transcatheter polytetrafluoroethylene-covered stent implantation in a giant CAAs with a $90 \%$ stenosis.

In our case, we used a combination of the treatment modalities outlined above. First, mechanical thrombus fragmentation was performed, and direct intracoronary thrombolysis using high doses of $r$ T-PA supported delineation of the vessel. Thrombus aspiration thereafter, in combination with additional local thrombolysis, outlined the exact anatomy of the RCA with large aneurysms and two severe stenoses, so that finally recanalization and complete reperfusion of the occluded RCA could be achieved by a combination of thrombectomy, intracoronary thrombolysis, and additional balloon angioplasty. PTCRA and DCA were not necessary due to the apparent lack of calcification.

\section{Conclusions}

Based on our experience and the literature presented, percutaneous coronary intervention is a safe and effective short-term treatment for children with acute myocardial infarction due to coronary sequelae of KD. The combination of various techniques may be necessary to address the different coronary pathologies as outlined in our case report.

\section{Acknowledgments}

This study was supported by hospital funding.

\section{Conflict of Interest}

The authors have no conflicts of interest relevant to this publication.

\section{Comment on this Article or Ask a Question}

\section{References}

1. Burns JC, Glodé MP. Kawasaki syndrome. Lancet. 2004;364:533-544. DOI: 10. 1016/S0140-6736(04)16814-1

2. Jang GY, Kang IS, Choi JY, Bae EJ, Kim YH, $\mathrm{Kim} \mathrm{SH}$, et al. Nationwide survey of coronary aneurysms with diameter $>6 \mathrm{~mm}$ in Kawasaki disease in Korea. Pediatr Int. 2014;57:367-372. DOI: 10.1111/ped.12536

3. Akagi T, Ogawa S, Ino T, Iwasa M, Echigo S, Kishida K, et al. Catheter interventional treatment in Kawasaki disease: a report from the Japanese Pediatric Interventional Cardiology Investigation group. J Pediatr. 2000;137:181-
186. DOI: $10.1067 / \mathrm{mpd} .2000 .107164$

4. Ariyoshi $M$, Shiraishi J, Kimura M, Matsui $A$, Takeda $M$, Arihara $M$, et al. Primary percutaneous coronary intervention for acute myocardial infarction due to possible sequelae of Kawasaki disease in young adults: a case series. Heart Ves. 2011;26:117-124. DOI: 10.1007/s00380010-0051-y

5. Kato H, Sugimura T, Akagi T, Sato N, Hashino $K$, Maeno $Y$, et al. Long-term consequences of Kawasaki disease. A 10- to 21-year follow-up study of 594 patients.
Circulation. 1996;94:1379-1385. DOI: 10.1161/01.CIR.94.6.1379

6. Harold JG, Bass TA, Bashore TM, Brindis RG, Brush JE Jr, Burke JA, et al. ACCF/ AHA/SCAI 2013 update of the clinical competence statement on coronary artery interventional procedures: a report of the American College of Cardiology Foundation/American Heart Association/ American College of Physicians Task Force on Clinical Competence and Training (writing committee to revise the 2007 clinical competence statement on car- 
diac interventional procedures). Circulation. 2013;128:436-472. DOI: 10.1161/ CIR.0b013e318299cd8a

7. Parsa SA, Khaheshi I, Paydary K, Haybar H. Acute myocardial infarction in a 35-yearold man with coronary artery aneurysm most probably caused by Kawasaki disease. Asian Pac J Trop Biomed. 2014;4:S50-S52. DOI: 10.12980/APJTB.4.2014C988

8. Rizk SR, El Said G, Daniels LB, Burns JC, El Said $\mathrm{H}$, Sorour KA, et al. Acute myocardial ischemia in adults secondary to missed Kawasaki disease in childhood. Am J Cardiol. 2015;115:423-427. DOI: 10.1016/j. amjcard.2014.11.024

9. Ishii $M$, Ueno $T$, Ikeda $H$, lemura $M$, Sugimura T, Furui J, et al. Sequential follow-up results of catheter intervention for coronary artery lesions after Kawasaki disease: Quantitative coronary artery angiography and intravascular ultrasound imaging study. Circulation. 2002;105:3004-3010. DOI: 10.1161/01. CIR.0000019733.56553.D8

10. Krendel S, Pollack P, Hanly J. Tissue plasminogen activator in pediatric myocardial infarction. Ann Emerg Med. 2000;35:502505. DOI: 10.1016/S0196-0644(00)70011-4

11. Levy DM, Silverman ED, Massicotte MP, McCrindle BW, Yeung RS. Long-term outcomes in patients with giant aneurysms secondary to Kawasaki disease. J Rheumatol. 2005;32:928-934. PMID: 15868632

12. Al-Mashham Y, Sinclair BG, Duncan WJ. Giant coronary arterial aneurysms and thrombosis in an infant with Kawasaki disease. Cardiol Young. 2006;16:510. DOI: 10.1017/S1047951106000849

13. Ishii M, Ueno T, Akagi T, Baba K, Harada K, Hamaoka K, et al. Guidelines for catheter intervention in coronary artery lesion in Ka- wasaki disease. Pediatr Int. 2001;43:558-562. DOI: 10.1046/j.1442-200X.2001.01464.x

14. Karia VR, Hescock GC, Gedalia A, Ross-Ascuitto N. Successful emergent coronary thrombolysis in a neonate with Kawasaki's disease. Pediatr Cardiol. 2010;31:12391242. DOI: $10.1007 / s 00246-010-9781-y$

15. Inaba S, Higaki T, Nagashima M, Nishimura K, Ogimoto A, Higaki J, et al. Successful revascularization by pulse infusion thrombolysis in a patient with Kawasaki disease combined with acute myocardial infarction. JACC Cardiovasc Interv. 2010;3:10911092. DOI: $10.1016 /$ j.jcin.2010.05.020

16. Mongiovì $M$, Alaimo $A$, Vernuccio $F$, Pieri D. Primary percutaneous coronary intervention for acute myocardial infarction in a pediatric patient with giant coronary aneurysm due to Kawasaki disease. Congenit Heart Dis. 2014;9:E16-E18. DOI: 10.1111/ chd. 12056

17. Sugimura T, Yokoi $H$, Sato $N$, Akagi T, Kimura $T$, lemura $M$, et al. Interventional treatment for children with severe coronary artery stenosis with calcification after long-term Kawasaki disease. Circulation. 1997;96:3928-3933. DOI: 10.1161/01. CIR.96.11.3928

18. Tsuda E, Miyazaki S, Yamada O, Takamuro M, Takekawa T, Echigo S. Percutaneous transluminal coronary rotational atherectomy for localized stenosis caused by Kawasaki disease. Pediatr Cardiol. 2006;27:447-453. DOI: 10.1007/s00246-006-1276-5

19. Tsuda E, Miyazaki S, Takamuro M, Fuse S, Tsuji Y, Echigo S. Strategy for localized stenosis caused by Kawasaki disease: Midterm results of percutaneous transluminal coronary balloon angioplasty in two infants. Pediatr Cardiol. 2006;27:272-275.
DOI: $10.1007 / \mathrm{s} 00246-005-1051-z$

20. Drossner DM, Chappell C, Rab T, Kim D. Percutaneous coronary intervention for acute myocardial infarction in a pediatric patient with coronary aneurysm and stenosis due to Kawasaki disease. Pediatr Cardiol. 2012;33:811-813. DOI: 10.1007/s00246012-0190-2

21. Dahdah N, Ibrahim R, Cannon L. First recanalization of a coronary artery chronic total obstruction in an 11-year-old child with Kawasaki disease sequelae using the CROSSER catheter. Pediatr Cardiol. 2007;28:389-393. DOI: 10.1007/s00246006-0083-3

22. Waki K, Arakaki Y, Mitsudo K. Long-term outcome of transcatheter polytetrafluoroethylene-covered stent implantation in a giant coronary aneurysm of a child with Kawasaki disease. Catheter Cardiovasc Interv. 2013;81:713-716. DOI: 10.1002/ ccd. 24486

Cite this article as: Meyer Z, Laser KT, Bach S, Hesse C, Kececioglu D, Scholtz W, Happel CM, NA Haas. Successful Revascularization of a Completely Occluded Right Coronary Artery by Local Thrombus Fragmentation, Thrombolysis, Thrombus Aspiration, and Balloon Angioplasty in a Patient with Atypical Kawasaki Disease: A Case Report and Review of the Literature. Structural Heart Disease. 2016;2(5):224-230. DOI: http:// dx.doi.org/10.12945/j.jshd.2016. 011.15 